Faculty of Mathematical Sciences

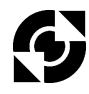

University of Twente

The Netherlands
P.O. Box 217

7500 AE Enschede

The Netherlands

Phone: +31-53-4893400

Fax: +31-53-4893114

Email: memo@math.utwente.nl

www.math.utwente.nl/publications

Memorandum No. 1601

Capacitated two-indenture models

for repairable item systems

W.H.M. ZIJM AND Z.M. AvșaR ${ }^{1}$

DeCEMBer 2001

ISSN 0169-2690

\footnotetext{
${ }^{1}$ Department of Industrial Engineering, Bilkent University, Bilkent 06533, Ankara, Turkey
} 


\title{
Capacitated Two-Indenture Models for Repairable Item Systems
}

\author{
W. Henk Zijm¹ and Zeynep Müge Avṣar² \\ (1) Faculty of Mathematical Sciences \\ University of Twente \\ P.O. Box 217, 7500 AE Enschede, The Netherlands \\ (2) Department of Industrial Engineering \\ Bilkent University, Bilkent 06533, Ankara, Turkey
}

November 30, 2001

\begin{abstract}
A two-indenture maintenance system is considered for a number of identical installations, in use at a single site. The installations are considered as assemblies that are made up of a number of repairable components. A component repair center and an assembly facility are both modeled as product form queuing networks. Failures of the assemblies occur according to a Poisson process. There are stock points for both ready-for-use assemblies and components. The inventory control policy employed at each stock point is a base-stock policy. Upon failure of an assembly, it is replaced by a ready-for-use one, if available. The failed assembly is inspected to identify and disassemble the broken component causing its failure. Next, the broken component is sent to the component repair center and one readyfor-use component is requested from the stock of this component. Once a ready-for-use component is available for the assembly, the request and the available component are merged and sent to the assembly facility. Completion of a component repair or an assembly operation results in a replenishment of the corresponding stock point. Requests that can not be satisfied right away are backordered. Service discipline is first-come-first-serve at each facility. Assuming that only one component is identified at a time as the cause of an assembly failure, first an alternative slightly aggregated (but exact) formulation is given for the system and then a near-product-form solution is proposed as an approximate steady-state distribution of the aggregated system. Comparison of the approximate performance measures computed with the use of the near-product-form solution and the ones obtained with simulation shows that the approximation is quite accurate. Relying on the accuracy, approximate performance measures are used for optimizing base stock levels with a greedy procedure.

Keywords: two-indenture systems, repairable items, capacitated servers
\end{abstract}

2000 Mathematical subject classification: 90B05, 90B15, 60K30

\section{Introduction}

Performance analysis of multi-indenture models with repairable items goes back to the MODMETRIC models due to Muckstadt[7]. MODMETRIC is an extension of the METRIC model developed by Sherbrooke[11] for multi-echelon inventory systems to the cases with complex hierarchical product structures. In the MODMETRIC model, the key assumptions are that the service facilities are uncapacitated and the failures of the items are Poisson from a population considered to be infinite, which make it possible to use Palm's theorem to analyze such systems. Later, Scudder and Hausman[4], [10], Gupta and Albright[3], Rustenburg[8] and Rustenburg et.al.[9] studied multi-indenture problems either to investigate system behaviour thoroughly or to approximate steady-state behaviour relaxing the quite restrictive assumptions of the MODMETRIC model. 
In this paper, capacitated two-indenture models are considered for the case of identical installations, that are modeled as assemblies with repairable components. Both the component repair center and the assembly facility are modeled as product form queuing networks. The goal of this paper is to develop accurate approximation procedures to determine a number of highly relevant performance measures (fill rate, work in process, stockout probability, expected shortage) of the capacitated two-indenture system. The organization of this article is as follows: In section 2, we outline in detail an approximation procedure, developed earlier for a two echelon system in [1], for a simple linear product structure with the repair and assembly centers modeled as single server facilities. Results of the numerical experiments are presented. Being convinced that the approximation is quite accurate, the next section is devoted to an extension of the approximation approach to the more realistic and complicated case with facilities being modeled as product form queuing networks. In Section 4, a greedy procedure is employed to optimize base stock levels in order to achieve a given target performance level under a budget constraint using (and relying on the accuracy of) the approximate values of the chosen performance measure to define a target performance level.

\section{Simple systems with single server facilities}

Consider a system with one type of assemblies in use at a site. Each assembly consists of a number (say, $L$ ) of repairable components. In Figure 1, such a system is depicted for the two-component case. Service facilities are for repair of broken components and for assembly operations. We assume that failures of assemblies occur according to a Poisson process with rate $\lambda$. Whenever an assembly fails, a request is generated for a ready-for-use assembly to replace the broken assembly at the site with a ready-for-use one from the assembly stock and the broken assembly is immediately inspected to identify and disassemble the component which causes failure of the assembly. As a result of an inspection component $l$ is identified as the cause of the failure with probability $\frac{\lambda_{l}}{\lambda}$ (we assume that always a single component causes the failure). The disassembled component is then sent to the component repair facility and the remaining body of the assembly requires a ready-for-use component of type $l$ to be satisfied from the corresponding stock point. If a request can not be satisfied immediately, it is backordered. At the stock points, base stock policies are employed for inventory control. $S_{0}$ and $S_{l}, l=1, \ldots, L$, are the target inventory levels for the assembly and the components of type $l$, respectively. So, initially there are $S_{0}\left(S_{l}\right)$ units of ready-for-use assemblies (components of type $l$ ) at the stock point of the assemblies (components of type $l$ ).

For simplicity of the first analysis and also for demonstration purposes in introducing the approximation approach, in this section each service facility is assumed to be a single exponential server. Relaxing this assumption (i.e., allowing service facilities to be product form queuing networks), a more general but complicated case is studied in Section 3. Furthermore, throughout the article it is assumed that inspectiondisassembly performed upon failure of an assembly is an instantaneous operation, again for the sake of having the notation and analysis of the model simple. The observations and the results in the article hold true for any queuing network that could be used to model an inspection-disassembly unit because there is not any synchronization (no stock point) following such an inspection-disassembly operation, which means behaviour of the departures from an inspection-disassembly unit to the component repair facility would be as in a tandem queue structure. Since the steady-state behaviour of tandem queues is known and our study is towards handling the difficulties arising due to synchronization at component stock points, the presentation in this article just to point out the arguments regarding synchronization could easily be extended to the case with the inspection-disassembly unit being represented as a network.

Now, in order to introduce notations under the simplifying assumptions stated in the previous paragraph, consider the model in Figure 1 eliminating inspection-disassembly unit and replacing the component repair and assembly networks with single exponential servers. $N_{l}$ is the random variable for the number of type $l$ components at the component repair facility. Also, let $\boldsymbol{N}=\left(N_{1}, \ldots, N_{L}\right)$ and let $|\boldsymbol{N}|$ denote the cardinality 
of $\boldsymbol{N}$, i.e., $\sum_{l=1}^{L} N_{l}$. Similarly, $M$ denotes the number of jobs to be processed at the assembly facility. $\bar{N}_{l}$ $(\bar{M})$ is the number of ready-for-use components of type $l$ (assemblies) in stock. $K_{0}$ and $K_{l}$ are the number of backordered requests for the assembly and component type $l$, respectively. Let $\boldsymbol{K}=\left(K_{1}, \ldots, K_{L}\right)$ and $|\boldsymbol{K}|=\sum_{l=1}^{L} K_{l}$.

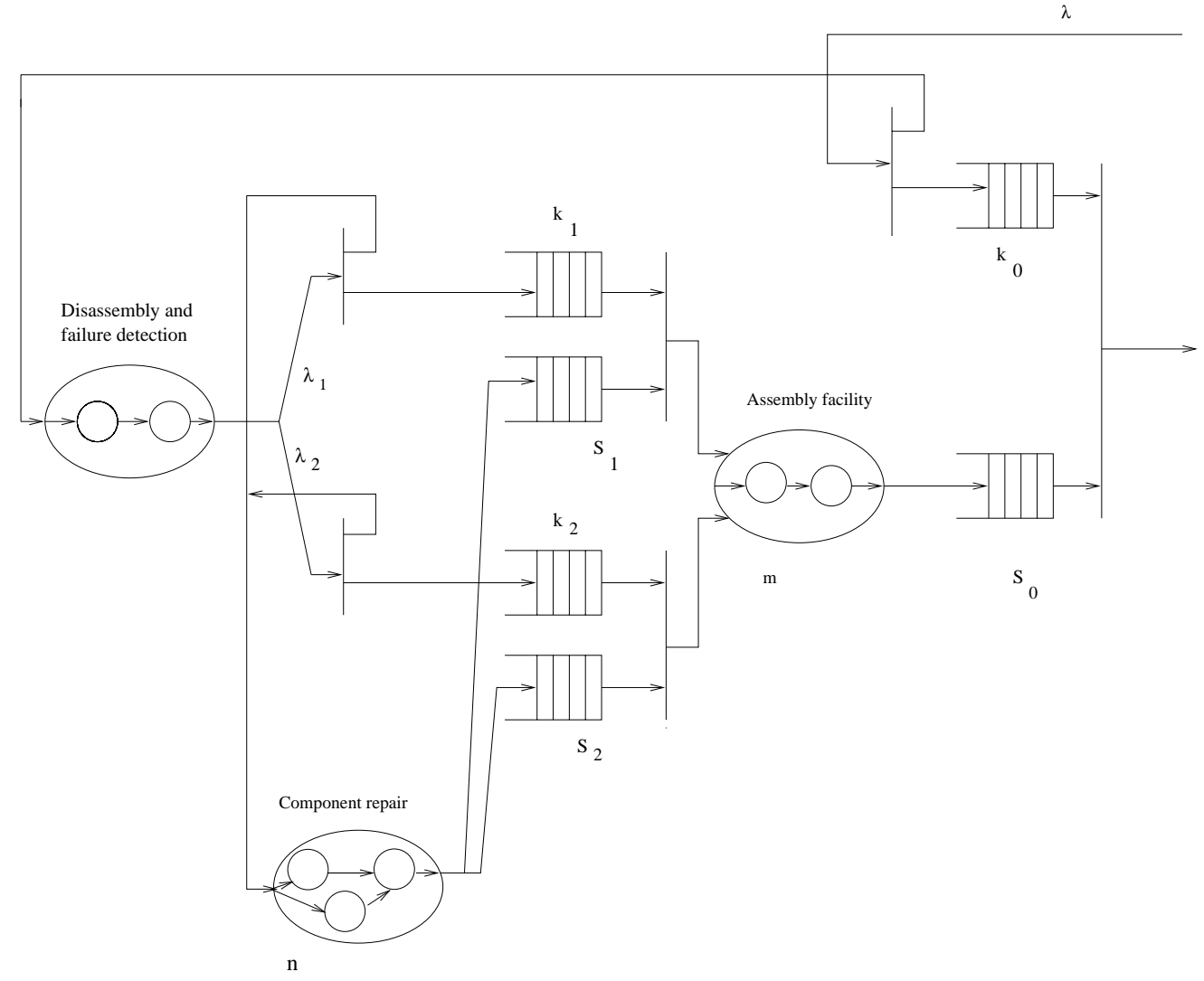

Figure 1: A two-indenture model with a single assembly and two components

The operating inventory control policies imply the following equations for any $\boldsymbol{N}=\boldsymbol{n}, \bar{N}_{l}=\bar{n}_{l}, M=m$, $\bar{M}=\bar{m}, \boldsymbol{K}=\boldsymbol{k}$ and $K_{0}=k_{0}$ :

$$
\begin{array}{r}
n_{l}+\bar{n}_{l}-k_{l}=S_{l} \quad \text { for } l=1, \ldots, L, \\
m+\bar{m}+|\boldsymbol{k}|-k_{0}=S_{0} .
\end{array}
$$

Also, as seen in Figure 1, whenever there is a request for an assembly (component), while at the same time a ready-for-use assembly (component) is available in the corresponding stock, those two are instantaneously merged to be sent to the site and to be used there instead of a broken assembly (to be assembled at the 
assembly facility), which means $\bar{n}_{l} \cdot k_{l}=0$ for $l=1, \ldots, L$ and $\bar{m} \cdot k_{0}=0$. Thus,

$$
\begin{aligned}
& \text { If } n_{l} \leq S_{l} \text {, then } \bar{n}_{l}=S_{l}-n_{l} \text { and } k_{l}=0 ; \\
& \text { If } n_{l}>S_{l} \text {, then } \bar{n}_{l}=0 \text { and } k_{l}=n_{l}-S_{l} ; \\
& \text { If } m+|\boldsymbol{k}| \leq S_{0} \text {, then } \bar{m}=S_{0}-(m+|\boldsymbol{k}|) \text { and } k_{0}=0 ; \\
& \text { If } m+|\boldsymbol{k}|>S_{0} \text {, then } \bar{m}=0 \text { and } k_{0}=(m+|\boldsymbol{k}|)-S_{0} .
\end{aligned}
$$

\subsection{The case of one critical component}

For the purpose of presenting the approximation approach, consider the simple two-indenture system in Figure 2 where $L=1$, i.e., each assembly is assumed to fail due to only one, so called critical, component, which means that other components either do not fail or are so cheap that they can be immediately replaced with ready-for-use spare components in stock. Hence, in the model only the stock point of the critical component is represented.

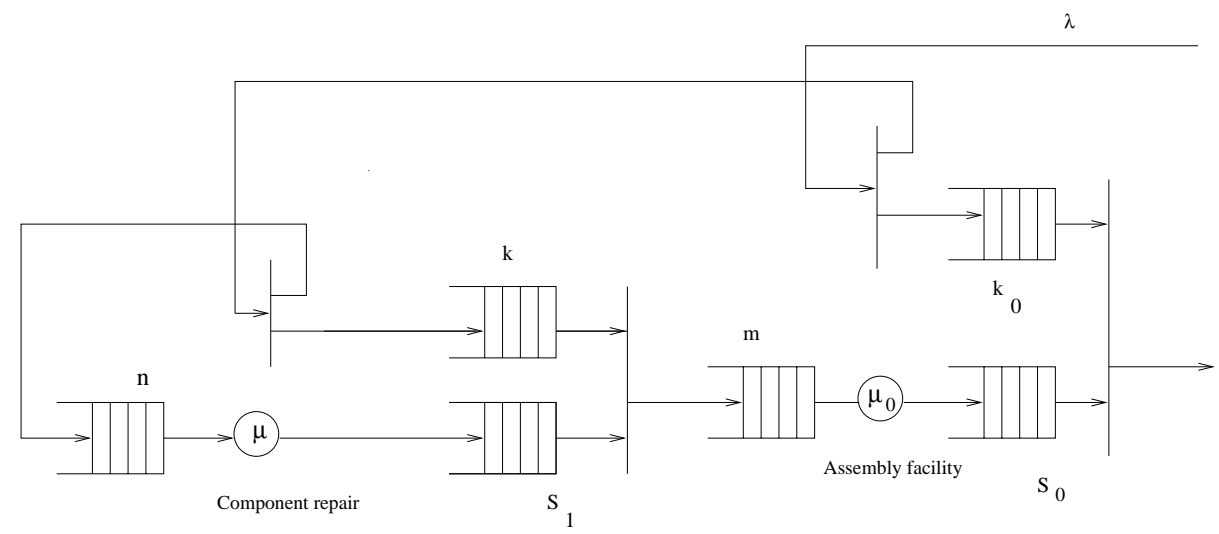

Figure 2: A two-indenture model with a single assembly and one critical component

The exponential service rate of the single server node of the component repair (assembly) facility is $\mu$ $\left(\mu_{0}\right)$. Since $\boldsymbol{N}$ and $\boldsymbol{K}$ both are one dimensional when $L=1$, denote $\boldsymbol{N}, \boldsymbol{K}, n$ and $k_{1}$ by $N, K, n$ and $k$, respectively. One possible state description for this system is $(n, m)$ and the corresponding state-transition diagram is seen in Figure 3. On the other hand, employment of the partial aggregation idea proposed in [1] for two-echelon capacitated repairable item systems leads to the alternative state description $(k, m)$ for the system analyzed here. This partial aggregation is due to representing the collection of the cases $n \leq S_{1}$ by $k=0$ (and each case $n>S_{1}$ by $k=n-S_{1}$ ). Denote the steady-state distribution of the model with state definition $(n, m)$ and $(k, m)$ by $P$ and $\tilde{P}$, respectively. Also, to keep the notation simple let $P(N=n, M=m)=P(n, m)$ and $\tilde{P}(K=k, M=m)=\tilde{P}(k, m)$. For any $k$ and $m$, the relation between the steady-state probabilities of the former (original) model and the partially aggregated model is

$$
\begin{aligned}
\tilde{P}(0, m) & =\sum_{n \leq S_{1}} P(n, m), \\
\tilde{P}(k, m) & =P\left(S_{1}+k, m\right) \quad \text { for } k>0 .
\end{aligned}
$$




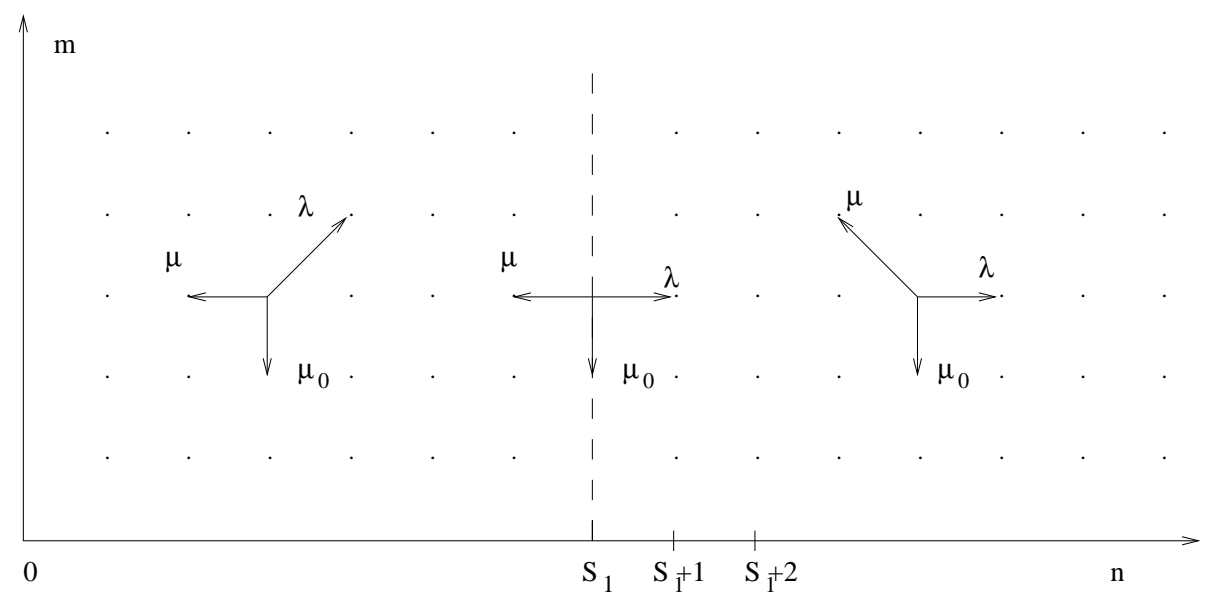

Figure 3: Transition diagram for state description $(n, m)$

Since there exists a one-to-one relationship between the states $(n, m)$ with $n>S_{1}$ of the original model, and the states $(k, m)$ with $k>0$ of the aggregated model, the transition rates in the interior state space in Figure 4 follow immediately. However, at the boundary $(0, m)$ of the aggregated model, we have to be more careful. Define $q(m)$ as the steady state probability that, given $m$ jobs in the assembly facility, an arriving component request has to wait (because stock of the component has already been depleted) although there is no other waiting component request present at the time of this arrival. Since Poisson arrivals see time averages (i.e., using PASTA property), we have

$$
q(m)=\frac{P\left(S_{1}, m\right)}{\sum_{n \leq S_{1}} P(n, m)} \quad \text { for every } m .
$$

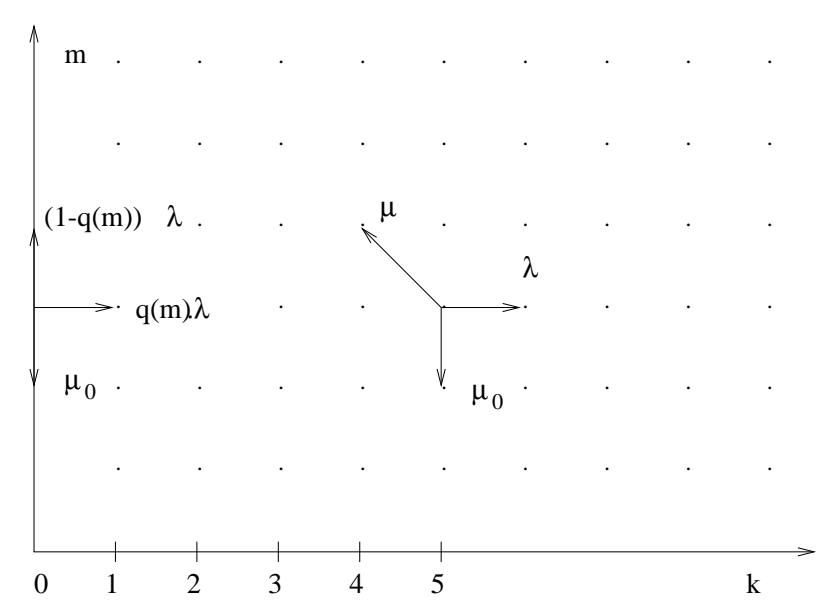

Figure 4: Transition diagram for state description $(k, m)$

It is now easy to verify that the full transition diagram of the aggregate model is given as in Figure 4 (see [12] for a formal proof). 
Next, the partially aggregated model with state description $(k, m)$ is approximated by ignoring the dependence of $q(m)$ on $m$ as in [1]. Hence, $q(m)$ is assumed to be equal to $q$ for every $m$. It can be easily seen that $q$ is, in fact, the expected probability that a critical component request is backordered although there is not any backorder of component request at the arrival time of this request, i.e.,

$$
q=\sum_{m=0}^{\infty} q(m) P\left(M=m \mid N \leq S_{1}\right) .
$$

The independence of $q$ from $m$ leads to a near-product-form solution given by Theorem 1 . We use the term near-product-form (instead of product form) due to partial aggregation. From this point on, this approximate model will be called the modified aggregate model. It should be pointed out that when $S_{1}=0$, there is no aggregation and the system behaves as a tandem queue for which the steady-state distribution is known to be of product-form. In other words, the approximation is exact in this case.

Theorem 1. The steady-state distribution of the modified aggregate model is

$$
\check{P}\left(K_{0}=k_{0}, M=m\right)= \begin{cases}P_{c}\left(N \leq S_{1}\right) P_{0}(M=m) & \text { for } k_{0}=0, \\ P_{c}\left(N=S_{1}+k\right) P_{0}(M=m) & \text { for } k_{0}>0,\end{cases}
$$

where $P_{c}(N=n)=(1-\rho) \rho^{n}$ with $\rho=(\lambda / \mu)<1$ and $P_{0}(M=m)=\left(1-\rho_{0}\right) \rho_{0}^{m}$ with $\rho_{0}=\left(\lambda / \mu_{0}\right)<1$.

Proof: The result follows immediately by replacing $q(m)$ 's in the balance equations of the exact aggregate model with $q$, which is equal to $\frac{P_{c}\left(N=S_{1}\right)}{P_{c}\left(N \leq S_{1}\right)}$.

The system studied in this section has been considered also by Lee and Zipkin[6] in the context of multiechelon systems to approximate the steady-state behaviour. Equivalence of the approximate steady-state distribution due to Lee and Zipkin in [6] and the near-product-form solution proposed by employing the $" q(m)$ to $q "$ argument has already been proven in [1]. For a detailed comparison of the assumption discussed in this paper, and the one in [6], the reader is referred to [1].

\subsection{Multi-component case}

In this section, the formulation and the corresponding analysis presented for $L=1$ in the previous section are extended to the multi-component case, and this way generalized for any $L \geq 1$.

Proceeding along the line of development in Section 2.1, $(\boldsymbol{n}, m)$ and the aggregate vector $(\boldsymbol{k}, m)$ are two alternative state descriptions. The aggregate model is fully expressed in terms of the former one by defining the following sets for every $\boldsymbol{k}$ :

$$
\begin{aligned}
& \mathcal{D}_{a}(\boldsymbol{k})=\left\{l \mid k_{l}=a\right\} \quad \text { for } \quad a=0,1, \ldots, \\
& \mathcal{T}(\boldsymbol{k})=\left\{\boldsymbol{n} \mid n_{d} \leq S_{d} \text { for } \quad d \in \mathcal{D}_{0}(\boldsymbol{k}), n_{d}=S_{d}+k_{d} \text { for } d \notin \mathcal{D}_{0}(\boldsymbol{k})\right\} .
\end{aligned}
$$

For any $\boldsymbol{k}$ and $m$, the steady-state probabilities of the partially aggregated model satisfy the following relation which is directly implied by the aggregation:

$$
\tilde{P}(\boldsymbol{k}, m)=\sum_{\boldsymbol{n} \in \mathcal{T}(\boldsymbol{k})} P(\boldsymbol{n}, m) .
$$

In the partially aggregated model, the steady-state probability that an arriving request for component $l$ waits although it finds no other waiting requests in front of it (i.e., there is not any backordered request 
but also no ready-for-use component of type $l$ at the corresponding stock point at the arrival time of this request) is now denoted by $q_{l}(\boldsymbol{k}, m)$ as a function of the vector $\boldsymbol{k}$ for every arriving request for type $\boldsymbol{l}$. Thus, $q_{l}(\boldsymbol{k}, m)$ is defined for every $\boldsymbol{k}$ with $k_{l}=0$, i.e., $l \in D_{0}(\boldsymbol{k})$, and every $M=m$ as follows:

$$
q_{l}(\boldsymbol{k}, m)=\frac{\sum_{\boldsymbol{n} \in \mathcal{T}_{l}(\boldsymbol{k})} P(\boldsymbol{n}, m)}{\sum_{\boldsymbol{n} \in \mathcal{T}(\boldsymbol{k})} P(\boldsymbol{n}, m)} \quad \text { for every } l \text { and } \boldsymbol{k} \ni k_{l}=0 \text {, and } m,
$$

where

$$
\mathcal{T}_{l}(\boldsymbol{k})=\left\{\boldsymbol{n} \mid n_{l}=S_{l} ; n_{d} \leq S_{d} \text { if } d \neq l, d \in \mathcal{D}_{0}(\boldsymbol{k}) ; n_{d}=S_{d}+k_{d} \text { if } d \notin \mathcal{D}_{0}(\boldsymbol{k})\right\} .
$$

Using arguments similar to the ones in Section 2.1, balance equations of the aggregate model can be obtained. Derivations to express transition rates of the aggregate model in terms of $q_{l}(\boldsymbol{k}, m)$ 's can be found in [12].

Analogous to the approximation in Section 2.1, the aggregate model with state description $(\boldsymbol{k}, m)$ studied in this section is approximated by assuming that $q_{l}(\boldsymbol{k}, m)$ 's used to express transition rates of the aggregate model are independent of $m$, i.e.,

$$
q_{l}(\boldsymbol{k})=\frac{P_{c}\left(\boldsymbol{N} \in \mathcal{T}_{l}(\boldsymbol{k})\right)}{P_{c}(\boldsymbol{N} \in \mathcal{T}(\boldsymbol{k}))} \quad \text { for every } l \text { and } \boldsymbol{k} \ni k_{l}=0,
$$

where $P_{c}$ is the marginal distribution for the random variable $N$ as defined below:

$$
P_{c}(\boldsymbol{N}=\boldsymbol{n})=\left((1-\rho) \rho^{|n|}\right)\left(\frac{\left(\sum_{l=1}^{L} n_{l}\right) !}{\left(\prod_{l=1}^{L} n_{l} !\right)} \prod_{l=1}^{L}\left(\frac{\lambda_{l}}{\lambda}\right)^{n_{l}}\right)
$$

with $(1-\rho) \rho^{|\boldsymbol{n}|}$ in the relation above being the steady state probability that $|\boldsymbol{N}|=|\boldsymbol{n}|$.

As in the previous section, $q_{l}(k)$ is again observed as the expected probability that a request of type $l$ has to wait although it arrives at a point in time there is no backordered request at the stock point of component $l$, i.e.,

$$
q_{l}(\boldsymbol{k})=\sum_{m=0}^{\infty} q_{l}(\boldsymbol{k}, m) P(M=m \mid \boldsymbol{N} \in \mathcal{T}(\boldsymbol{k})) .
$$

Note that the approximation is exact when $S_{l}=0$ for every component $l$.

Then, the near-product-form solution for the modified aggregate model is as in Theorem 2. One would immediately recognize that this solution is, in fact, nothing but a generalization of the one given in Theorem 1 allowing for the modeling of multiple component types. The proof is skipped to keep the presentation simple, interested readers are referred to [12].

Theorem 2. The steady-state distribution of the modified aggregate model is

$$
\check{P}(\boldsymbol{K}=\boldsymbol{k}, M=m)=P_{c}(\boldsymbol{N} \in \mathcal{T}(\boldsymbol{k})) P_{0}(M=m) .
$$

As pointed out in [1] for two-echelon systems which are equivalent to the ones studied in Section 2.1, note that the stronger assumption that $\boldsymbol{N}$ and $M$ are totally independent, and Poisson arrivals (with rate $\lambda$ ) for the assembly facility, imply the same approximation of $P(\boldsymbol{n}, m)$ by $P_{c}(\boldsymbol{N}=\boldsymbol{n}) P_{0}(M=m)$, and then using (5) the same near-product-form solution is obtained. On the other hand, the " $q_{l}(\boldsymbol{k}, m)$ to $q_{l}(\boldsymbol{k})$ " argument is assuming only $P\left(\boldsymbol{N} \in \mathcal{T}_{l}(\boldsymbol{k}), M=m\right)=P_{c}\left(\boldsymbol{N} \in \mathcal{T}_{l}(\boldsymbol{k})\right) P_{0}(M=m)$ for every aggregation represented by vector $\boldsymbol{k}$ such that $k_{l}=0$ and $P(\boldsymbol{N} \in \mathcal{T}(\boldsymbol{k}), M=m)$ is equal to $P_{c}(\boldsymbol{N} \in \mathcal{T}(\boldsymbol{k})) P_{0}(M=m)$. 


\subsection{Performance of the approximation}

For the performance evaluation of the single assembly, two-indenture model studied in Section 2.2 for the case of two components, we consider four performance measures for the assembly facility, namely, the stockout probability $(S P)$, the fill rate $(F R)$, the expected shortage $(E S)$ of the assemblies, and the expected work in process inventory $(E W)$ defined as

$$
\begin{aligned}
S P & =\operatorname{Pr}\left(K_{0}>0\right), \\
F R & =\operatorname{Pr}\left(|\boldsymbol{K}|+M<S_{0}\right)=\operatorname{Pr}\left(K_{0}=0\right)-\operatorname{Pr}\left(|\boldsymbol{K}|+M=S_{0}\right), \\
E S & =E\left(K_{0}\right), \\
E W & =E(|\boldsymbol{K}|+\boldsymbol{M}) .
\end{aligned}
$$

The key observation is that the distribution of the random variable $K_{0}$ follows easily from the near-product property of the distribution of $(\boldsymbol{k}, m)$, as follows:

$$
\begin{aligned}
\operatorname{Pr}\left(K_{0}=0\right) & =\sum_{|\boldsymbol{k}|+m \leq S_{0}} \tilde{P}(\boldsymbol{k}, m), \\
\operatorname{Pr}\left(K_{0}=k_{0}\right) & =\sum_{|\boldsymbol{k}|+m=S_{0}+k_{0}} \tilde{P}(\boldsymbol{k}, m) \quad \text { for } k_{0}>0 .
\end{aligned}
$$

The fill rate is the probability that any demand arriving can be satisfied immediately from the assembly stock. The work in process inventory considered here is the total number of backorders at the component stock points, i.e., $k_{1}+k_{2}$, and the number being (or to be) served at the assembly facility, i.e., $m$. Note that $E W$ does not depend on $S_{0}$, which makes $E W$ a measure to evaluate the performance of the approximation without trying different $S_{0}$ values.

For the purpose of observing how accurate the proposed approximation is, these performance measures computed using simulation are compared with the ones derived by the near-product-form distribution found as the solution to the modified aggregate model. The simulation results given are the averages of the performance measures obtained by 15 replications. The confidence level for the confidence intervals constructed is 0.95. Tables 1 and 2 are for the case the failure rates $\lambda_{1}$ and $\lambda_{2}$ both being 4 , and $\mu=\mu_{0}=10$. Tables 4 and 5 in the appendix are for the case $\lambda_{1}=8, \lambda_{2}=1, \mu=10, \mu_{0}=12$. As can be observed by going over the experiments in the tables, the cases considered are to get an insight into how sensitive the performance measures are to changes in target inventory levels of the components and the assembly. (Although results of the numerical experiments are presented only for the performance measures EW and FR to have the article in a compact form, the experiments are all to compare the approximations for four performance measures listed above with the simulation results and the comments made following this sentence are based on a thorough analysis of all of these comparisons.) Relative error percentages are quite low in general, the highest error observed is around 7 percent, but approximate measures are not covered by the confidence intervals in most of the cases. Approximate measures fall in the confidence intervals when $S_{1}$ and $S_{2}$ values are both equal to 0 (which is the case where the approximation is exact, differences between simulation and approximation values observed beyond the third decimal digit for such cases must be due to rounding) or they are both large. In accordance with this observation, relative errors are low for $S_{1}, S_{2}$ values being very low (around zero) and they tend to increase first as $S_{1}$ and $S_{2}$ increase, and to decrease beyond some sufficiently large $S_{1}$ and $S_{2}$ values which depend on the system parameters. The former case could be explained by the closeness of the system behaviour to tandem queues (recall the exactness of the approximation when $S_{1}=S_{2}=0$ ). As for the latter case, stockout occurs less at the component stock points, which results in the arriving requests to be immediately merged with an available ready-for-use component and to be sent to the assembly facility with higher probability. In other words, the system behaves as if the two stages are independent with higher probability as $S_{1}$ and $S_{2}$ increase. 
Table 1: EW for $\rho=\rho_{0}=8 / 10$

\begin{tabular}{|c|c|c|c|c|c|}
\hline$\left(S_{1}, S_{2}\right)$ & $E W_{\text {sim }}$ & Conf.Interval & Std.Dev.EW & $E W_{a p p}$ & error $_{E W}$ \\
\hline$\overline{(\overline{(0,0)}}$ & 8.00355 & ("(7.98754,8.01957) & $\overline{0.016}$ & 8.00000 & $\overline{0.04}$ \\
\hline$(1,1)$ & 6.50597 & $(6.49097,6.52097)$ & 0.007 & 6.66667 & 2.47 \\
\hline$(3,3)$ & 4.96101 & $(4.94834,4.97369)$ & 0.006 & 5.18519 & 4.52 \\
\hline$(5,5)$ & 4.37739 & $(4.36626,4.38851)$ & 0.005 & 4.52675 & 3.41 \\
\hline$(7,7)$ & 4.15210 & $(4.14145,4.16275)$ & 0.005 & 4.23411 & 1.98 \\
\hline$(9,9)$ & 4.06261 & $(4.05208,4.07315)$ & 0.005 & 4.10405 & 1.02 \\
\hline$(15,15)$ & 4.00531 & $(3.99473,4.01589)$ & 0.005 & 4.00913 & 0.10 \\
\hline$(1,0)$ & $\overline{77.24424}$ & 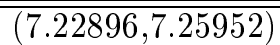 & $\overline{0.007}$ & 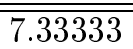 & $\overline{1.23}$ \\
\hline$(3,0)$ & 6.40109 & $(6.38701,6.41516)$ & 0.007 & 6.59259 & 2.99 \\
\hline$(5,0)$ & 6.04539 & $(6.03176,6.05902)$ & 0.006 & 6.26337 & 3.61 \\
\hline$(7,0)$ & 5.89487 & $(5.88205,5.90769)$ & 0.006 & 6.11706 & 3.77 \\
\hline$(9,0)$ & 5.83243 & $(5.81996,5.84489)$ & 0.006 & 6.05202 & 3.77 \\
\hline$(15,0)$ & 5.78959 & $(5.77705,5.80214)$ & 0.006 & 6.00457 & 3.71 \\
\hline$(1,2)$ & 6.01561 & (6.00154,6.02969) & $\overline{0.007}$ & 6.22222 & $\overline{3.43}$ \\
\hline$(3,2)$ & 5.24733 & $(5.23406,5.26061)$ & 0.006 & 5.48148 & 4.46 \\
\hline$(5,2)$ & 4.92202 & $(4.90951,4.93454)$ & 0.006 & 5.15226 & 4.68 \\
\hline$(7,2)$ & 4.78333 & $(4.77139,4.79528)$ & 0.006 & 5.00594 & 4.65 \\
\hline$(9,2)$ & 4.72363 & $(4.71191,4.73535)$ & 0.005 & 4.94091 & 4.60 \\
\hline$(15,2)$ & 4.68196 & $(4.67021,4.69371)$ & 0.005 & 4.89346 & 4.52 \\
\hline$(1,4)$ & 5.48654 & 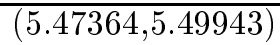 & 0.006 & 5.72840 & 4.41 \\
\hline$(3,4)$ & 4.77522 & $(4.76279,4.78766)$ & 0.006 & 4.98768 & 4.45 \\
\hline$(5,4)$ & 4.48520 & $(4.47343,4.49696)$ & 0.005 & 4.65844 & 3.86 \\
\hline$(7,4)$ & 4.36234 & $(4.35139,4.37330)$ & 0.005 & 4.51212 & 3.43 \\
\hline$(9,4)$ & 4.30846 & $(4.29740,4.31952)$ & 0.005 & 4.44709 & 3.22 \\
\hline$(15,4)$ & 4.27004 & $(4.25898,4.28110)$ & 0.005 & 4.39963 & 3.03 \\
\hline$\overline{(1,6)}$ & 5.26269 & 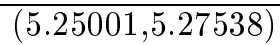 & 0.006 & 5.50892 & 4.68 \\
\hline$(3,6)$ & 4.57528 & $(4.56358,4.58697)$ & 0.005 & 4.76818 & 3.03 \\
\hline$(5,6)$ & 4.30646 & $(4.29558,4.31735)$ & 0.005 & 4.43896 & 3.08 \\
\hline$(7,6)$ & 4.19462 & $(4.18387,4.20538)$ & 0.005 & 4.29264 & 2.34 \\
\hline$(9,6)$ & 4.14632 & $(4.13560,4.15704)$ & 0.005 & 4.22761 & 1.96 \\
\hline$(15,6)$ & 4.11126 & $(4.10047,4.12206)$ & 0.005 & 4.18015 & 1.68 \\
\hline $\begin{array}{l}(1,20) \\
\end{array}$ & 5.09906 & (5.08678,5.11134) & $\overline{0.006}$ & 5.33393 & 4.61 \\
\hline$(3,20)$ & 4.42364 & $(4.41239,4.43489)$ & 0.005 & 4.59319 & 3.83 \\
\hline$(5,20)$ & 4.16951 & $(4.15900,4.18002)$ & 0.005 & 4.26398 & 2.27 \\
\hline$(7,20)$ & 4.07021 & $(4.05971,4.08071)$ & 0.005 & 4.11766 & 1.17 \\
\hline$(9,20)$ & 4.02990 & $(4.01939,4.04042)$ & 0.005 & 4.05263 & 0.56 \\
\hline$(15,20)$ & 4.00327 & $(3.99269,4.01384)$ & 0.005 & 4.00517 & 0.05 \\
\hline
\end{tabular}


Table 2: Fill Rate for $\rho=\rho_{0}=8 / 10$

\begin{tabular}{|c||c|c|c|}
\hline$\left(S_{0}, S_{1}, S_{2}\right)$ & $F R_{\text {sim }}$ & $F R_{\text {app }}$ & error $_{F R}$ \\
\hline \hline$(16,0,0)$ & 0.88164 & 0.88178 & 0.02 \\
\hline \hline$(15,2,0)$ & 0.89637 & 0.89360 & 0.31 \\
\hline$(15,1,1)$ & 0.89836 & 0.89614 & 0.25 \\
\hline \hline$(14,4,0)$ & 0.89919 & 0.89404 & 0.57 \\
\hline$(14,3,1)$ & 0.90595 & 0.90144 & 0.50 \\
\hline$(14,2,2)$ & 0.90791 & 0.90367 & 0.47 \\
\hline \hline$(13,6,0)$ & 0.89129 & 0.88441 & 0.77 \\
\hline$(13,5,1)$ & 0.90323 & 0.89687 & 0.70 \\
\hline$(13,4,2)$ & 0.90885 & 0.90321 & 0.62 \\
\hline$(\mathbf{1 3 , 3 , 3})$ & $\mathbf{0 . 9 1 0 4 8}$ & $\mathbf{0 . 9 0 5 1 4}$ & 0.59 \\
\hline \hline$(12,8,0)$ & 0.87370 & 0.86555 & 0.93 \\
\hline$(12,7,1)$ & 0.89111 & 0.88338 & 0.87 \\
\hline$(12,6,2)$ & 0.9007 & 0.89386 & 0.76 \\
\hline$(12,5,3)$ & 0.90530 & 0.89926 & 0.67 \\
\hline$(12,4,4)$ & 0.90665 & 0.90091 & 0.63 \\
\hline \hline$(11,10,0)$ & 0.84746 & 0.83802 & 1.11 \\
\hline$(11,9,1)$ & 0.87054 & 0.86153 & 1.03 \\
\hline$(11,8,2)$ & 0.88421 & 0.87628 & 0.90 \\
\hline$(11,7,3)$ & 0.89174 & 0.88504 & 0.75 \\
\hline$(11,6,4)$ & 0.89540 & 0.88959 & 0.65 \\
\hline$(11,5,5)$ & 0.89649 & 0.89099 & 0.61 \\
\hline
\end{tabular}

\section{The system with product form service facilities}

The results obtained in Section 2 for the simple system with single server facilities are generalized in this section by modeling the component repair and assembly facilities as product form open queuing networks with $J$ and $Z$ nodes, respectively (where a node may represent a number of parallel exponential servers). Before starting the analysis of this general model, the notation is adapted to fully describe the complexities introduced with the open networks. $N_{l}=\left(N_{l 1}, \ldots, N_{l J}\right)$ is the random vector for the number of type $l$ components at each node of the queuing network representing the component repair facility. Also, define $\boldsymbol{N}$ as $\left(\boldsymbol{N}_{1}, \ldots, \boldsymbol{N}_{L}\right)$. Similarly, $\boldsymbol{M}=\left(M_{1}, \ldots, M_{Z}\right)$ is the state vector defined for the assembly network to denote the number of assembly operations to be completed at each node of this network. $\bar{N}_{l}$ and $\bar{M}$, and $\boldsymbol{K}$ and $K_{0}$ are all as defined before. $p_{i j}^{(l)}$ and $\bar{p}_{y z}$ are the routing probabilities from node $i$ to $j$ for component of type $l$ and from node $y$ to $z$ within the component repair and the assembly networks, respectively. $p_{0 j}^{(l)}$ and $\bar{p}_{0 z}\left(p_{j 0}^{(l)}\right.$ and $\left.\bar{p}_{z 0}\right)$ are the routing probabilities of entering (leaving) the networks.

For any $\boldsymbol{N}_{l}=\boldsymbol{n}_{l}$ and $\bar{N}_{l}=\bar{n}_{l}$ for $l=1, \ldots, L, \boldsymbol{M}=\boldsymbol{m}, \bar{M}=\bar{m}, \boldsymbol{K}=\boldsymbol{k}$ and $K_{0}=k_{0}$, the equations implied by the operating inventory control policies are such that $n_{l}$ and $m$ in (1), (2) are replaced with $\left|\boldsymbol{n}_{l}\right|$ and $|\boldsymbol{m}|$, respectively. The state of the system is fully described by $(\boldsymbol{n}, \boldsymbol{m})$. For the alternative aggregate model, the state description is $(\boldsymbol{k}, \boldsymbol{m})$. The sets $\mathcal{D}_{a}(\boldsymbol{k})$ and $\mathcal{T}(\boldsymbol{k})$ and $\mathcal{T}_{l}(\boldsymbol{k})$ are as in Section 2.2 except that $\left|\boldsymbol{n}_{l}\right|$ and $\left|\boldsymbol{n}_{d}\right|$ are considered instead of $n_{l}$ and $n_{d}$, respectively, in defining $\mathcal{T}(\boldsymbol{k})$ and $\mathcal{T}_{l}(\boldsymbol{k})$.

$$
\tilde{P}(\boldsymbol{k}, \boldsymbol{m})=\sum_{\boldsymbol{n} \in \mathcal{T}(\boldsymbol{k})} P(\boldsymbol{n}, \boldsymbol{m}) .
$$

(Note that the dimension of the state space of the aggregate model may be significantly smaller than that of 
the original model.) Generalizing the development in the previous section, transition rates of the aggregate model are determined using the probability that an arrival of a broken assembly with a request for component $l$ is backordered although there is not any backordered request of type $l$ at the time this request arrives, i.e., $q_{l}(\boldsymbol{k}, \boldsymbol{m})$ for every $l$ and $\boldsymbol{k}$ such that $k_{l}=0$, and every $\boldsymbol{m}$.

As for the discussion of the approximate (modified aggregate) model, the notation of the previous section is revised for steady-state distributions of the networks in the model. Total arrival rates $\hat{\lambda}_{j}^{(l)}$ for the network of component repair and $\bar{\lambda}_{z}$ for the assembly network satisfy the following equations:

$$
\begin{aligned}
& \hat{\lambda}_{j}^{(l)}=\lambda_{l} p_{0 j}^{(l)}+\sum_{i=1}^{J} \hat{\lambda}_{i}^{(l)} p_{i j}^{(l)} \quad \text { for } j=1, \ldots, J \text { and } l=1, \ldots, L . \\
& \bar{\lambda}_{z}=\lambda \bar{p}_{0 z}+\sum_{y=1}^{Z} \bar{\lambda}_{y} \bar{p}_{y z} \quad \text { for } z=1, \ldots, Z .
\end{aligned}
$$

The distribution $P_{c}$ is defined as a product form solution in terms of $P_{c j}$ 's corresponding to the nodes of the component repair network as shown below:

$$
P_{c}(\boldsymbol{N}=\boldsymbol{n})=\prod_{j=1}^{J} P_{c j}\left(\sum_{l=1}^{L} N_{l j}=\sum_{l=1}^{L} n_{l j}\right)\left(\frac{\left(\sum_{l=1}^{L} n_{l j}\right) !}{\prod_{l=1}^{L} n_{l j} !} \prod_{l=1}^{L}\left(\frac{\hat{\lambda}_{j}^{(l)}}{\sum_{d=1}^{L} \hat{\lambda}_{j}^{(d)}}\right)^{n_{l j}}\right)
$$

with $P_{c j}\left(\sum_{l=1}^{L} N_{l j}=\sum_{l=1}^{L} n_{l j}\right)=\frac{\psi_{j}\left(\sum_{d=1}^{L} n_{j j}\right)}{\sum_{u=0}^{\infty} \psi_{j}(u)}$ for $\left(\sum_{l=1}^{L} n_{l j}\right)=0,1, \ldots$, where $\psi_{j}(0)=1$ and $\psi_{j}(\eta)=$ $\frac{\left(\sum_{l=1}^{L} \hat{\lambda}_{j}^{(l)}\right)^{\eta}}{\prod_{\zeta=1}^{\eta} \mu_{j}(\zeta)}$ for $\eta=1,2, \ldots$. As noted in the previous section, $P_{c}$ is in fact the marginal distribution for the component repair facility. Also, define a product form solution, namely $P_{0}(\boldsymbol{M}=\boldsymbol{m})$, as $\prod_{z=1}^{Z} P_{z}\left(M_{z}=m_{z}\right)$, where $P_{z}\left(M_{z}=m_{z}\right)=\frac{\psi_{0 z}\left(m_{z}\right)}{\sum_{\eta=0}^{\infty} \psi_{0 z}(\eta)}$ for $m_{z}=0,1, \ldots$, where $\psi_{0 z}(0)=1$ and $\psi_{0 z}(\eta)=\frac{\left(\bar{\lambda}_{z}\right)^{\eta}}{\prod_{\zeta=1}^{\eta} \mu_{z}(\zeta)}$ for $\eta=1,2, \ldots$. Readers less familiar with steady-state distributions for multi-class networks are referred to Buzacott and Shanthikumar[2] for an elaborate discussion of these models in the context of manufacturing systems.

As in Section 2, the proposed approximation is based on the assumption that the $q_{l}$ values are independent of $\boldsymbol{m}$, i.e., and hence to replace $q_{l}(\boldsymbol{k}, \boldsymbol{m})$ in the aggregate model with $q_{l}(\boldsymbol{k})$, which is as defined (with the revision of the notation) in Section 2. Then, the approximate near-product-form steady-state distribution given in Theorem 3 (proof is skipped, see [12]) is obtained as a solution to the modified (approximated) aggregate model.

Theorem 3. The steady-state distribution of the modified aggregate model is

$$
\check{P}(\boldsymbol{K}=\boldsymbol{k}, \boldsymbol{M}=\boldsymbol{m})=P_{c}(\boldsymbol{N} \in \mathcal{T}(\boldsymbol{k})) P_{0}(\boldsymbol{M}=\boldsymbol{m}) .
$$

Consider the special case such that each machine at the component repair facility is dedicated to only one type of component keeping all other characteristics defining the system as in this section. From analytical point of view, this is not any different from having product form component repair facilities as many as the number of components, each repairing only one component type. In this case, $P_{c}(\boldsymbol{N}=\boldsymbol{n})$ takes the simpler form $\prod_{l=1}^{L} \prod_{j \in \mathcal{J}_{l}} P_{c j}\left(N_{l j}=n_{l j}\right)=\prod_{l=1}^{L} P_{c}^{l}\left(\boldsymbol{N}_{l}=\boldsymbol{n}_{l}\right)$, where $\mathcal{J}_{l}$ is the set of machines used for the repair of (only) components of type $l$ and $P_{c}^{l}$ is the marginal product form probability distribution for the component repair network dedicated to component type $l$. Then, $P_{c}(\boldsymbol{N} \in \mathcal{T}(\boldsymbol{k}))$ that appears in the approximate near product form steady state distribution presented by Theorem 3 turns out to be $\prod_{l=1}^{L} P_{c}^{l}\left(\boldsymbol{N}_{l} \in \mathcal{T}^{l}\left(\boldsymbol{k}_{l}\right)\right)$, defining $\mathcal{T}^{l}\left(\boldsymbol{k}_{l}\right)$ as $\left\{\boldsymbol{n}_{l}|\quad| \boldsymbol{n}_{l} \mid \leq S_{l}\right.$ for $k_{l}=0,\left|\boldsymbol{n}_{l}\right|=S_{l}+k_{l}$ for $\left.k_{l}>0\right\}$ for all $l=1, \ldots, L$. Note that $\mathcal{T}(\boldsymbol{k})=\bigcup_{l=1}^{L} \mathcal{T}^{l}\left(\boldsymbol{k}_{l}\right)$. 


\section{Determining base stock levels to optimize assembly fill rate}

Based on the observation that the proposed approximate near-product-form solution leads to quite accurate performance measures for single-assembly, two-indenture models, in this section this approximation is used to set up a greedy procedure that aims at maximizing the overall system performance, given a stock investment amount. With the optimal system performance, we also obtain the optimal stock allocation, i.e., the values of $S_{l}$ for $l=0,1, \ldots, L$.

The performance measure considered here is the fill rate for the assembly, defined as $\operatorname{Pr}\left(|\boldsymbol{K}|+M<S_{0}\right)$. Since $\boldsymbol{K}$ is a function of $S_{1}, S_{2}, \ldots, S_{L}$, we may write $F R\left(S_{0}, S_{1}, S_{2}, \ldots, S_{L}\right)=\operatorname{Pr}\left(|\boldsymbol{K}|+M<S_{0}\right)$. Define $c_{l}$ as the investment associated with having one stock keeping unit (SKU) of item $l, l=0,1, \ldots L$, at the corresponding stock point (hence, including the assembly as well as all components). Furthermore, let $C$ be the allowed total investment. Then the (non-linear) optimization problem considered in this section can be written as

$$
\max \operatorname{FR}\left(S_{0}, S_{1}, S_{2}, \ldots, S_{L}\right)
$$

subject to

$$
\begin{aligned}
\sum_{l=0}^{L} c_{l} S_{l} & \leq C \\
S_{l} & \geq 0, \quad l=0,1, \ldots L .
\end{aligned}
$$

Note that this problem bears some similarity with a non-linear knapsack problem. Since analytical expressions for the fill rate become extremely complex, we instead use the following greedy procedure to approximate the optimal solution of the above optimization problem.

\section{Greedy Procedure}

Initialization:

Set $S_{l}=0$, for $l=0,1,2, \ldots, L$, and also set $\hat{C}=0$.

Improvement Step:

Define $\Delta_{l}$ for $l=0,1,2, \ldots L$, by

$$
\Delta_{l}= \begin{cases}\frac{F R\left(S_{0}, S_{1}, \ldots, S_{l}+1, \ldots, S_{L}\right)-F R\left(S_{0}, S_{1}, S_{2}, \ldots, S_{l}, \ldots, S_{L}\right)}{c_{l}} & \text { if } \hat{C}+c_{l} \leq C, \\ 0 & \text { otherwise. }\end{cases}
$$

If $\max _{l=0,1, \ldots, L}\left\{\Delta_{l}\right\}=0$, then STOP. Otherwise, let $\hat{l}=\arg \max _{l=0,1, \ldots L}\left\{\Delta_{l}\right\}$ and set $\hat{C}=\hat{C}+c_{\hat{l}}$, and then repeat the improvement step with $\left(S_{0}, S_{1}, \ldots, S_{\hat{l}}+1, \ldots, S_{L}\right)$.

As for the implementation of this greedy procedure, the fill rates used through iterations are all approximated by the near-product-form steady state probability distribution. The finally resulting value of $F R\left(S_{0}, S_{1}, S_{2}, \ldots, S_{L}\right)$ is used as an approximation for the optimum of the non-linear maximization problem defined above. Without going into details, we note that there are basically two reasons why this greedy procedure is expected to perform reasonably well: the already noted similarity with a knapsack problem and the fact that $\operatorname{FR}\left(S_{0}, S_{1}, S_{2}, \ldots, S_{L}\right)$ tends to behave as a multi-dimensional concave function in all its arguments, provided that $S_{0}$ is not too small. Note that at each execution of the improvement step we select one base stock value to increase by one, such that we obtain the maximum increase in fill rate per unit of money invested. This is called the "biggest bang for the buck" approach (see also Sherbrooke[11]). 
Table 3: Greedy approach for $\rho=\rho_{0}=8 / 10$

\begin{tabular}{|c|c|c|c|c|}
\hline$\left(S_{0}, S_{1}, S_{2}\right)$ & $F R_{a p p}$ & $\Delta_{0}$ & $\Delta_{1}$ & $\Delta_{2}$ \\
\hline$(0,0,0)$ & 0 & $\mathbf{0 . 0 2 0 0 0}$ & 0 & 0 \\
\hline$(1,0,0)$ & 0.04000 & $\mathbf{0 . 0 3 2 0 0}$ & 0.01600 & 0.01600 \\
\hline$(2,0,0)$ & 0.10400 & $\mathbf{0 . 0 3 8 4 0}$ & 0.03200 & 0.03200 \\
\hline$(3,0,0)$ & 0.18080 & 0.04096 & $\mathbf{0 . 0 4 3 5 2}$ & 0.04352 \\
\hline$(3,1,0)$ & 0.22432 & 0.04429 & 0.02355 & $\mathbf{0 . 0 5 2 7 4}$ \\
\hline$(3,1,1)$ & 0.27706 & $\mathbf{0 . 0 4 5 6 7}$ & 0.03052 & 0.03052 \\
\hline$(4,1,1)$ & 0.36840 & $\mathbf{0 . 0 4 2 0 2}$ & 0.03400 & 0.03400 \\
\hline$(5,1,1)$ & 0.45245 & $\mathbf{0 . 0 3 7 9 1}$ & 0.03516 & 0.03516 \\
\hline$(6,1,1)$ & 0.52827 & 0.03372 & 0.03464 & $\mathbf{0 . 0 3 4 6 4}$ \\
\hline$(6,1,2)$ & 0.56291 & 0.03290 & $\mathbf{0 . 0 3 4 9 9}$ & 0.02251 \\
\hline$(6,2,2)$ & 0.59790 & $\mathbf{0 . 0 3 1 5 6}$ & 0.02300 & 0.02300 \\
\hline$(7,2,2)$ & 0.66103 & $\mathbf{0 . 0 2 7 0 0}$ & 0.02175 & 0.02175 \\
\hline$(8,2,2)$ & 0.71503 & $\mathbf{0 . 0 2 2 9 9}$ & 0.02011 & 0.02011 \\
\hline$(9,2,2)$ & 0.76101 & $\mathbf{0 . 0 1 9 5 0}$ & 0.01827 & 0.01827 \\
\hline$(10,2,2)$ & 0.80000 & $\mathbf{0 . 0 1 6 4 8}$ & 0.01637 & 0.01637 \\
\hline$(11,2,2)$ & 0.83296 & 0.01389 & $\mathbf{0 . 0 1 4 5 0}$ & 0.01450 \\
\hline$(11,3,2)$ & 0.84746 & 0.01300 & 0.01033 & $\mathbf{0 . 0 1 3 3 3}$ \\
\hline$(11,3,3)$ & 0.86079 & $\mathbf{0 . 0 1 2 1 2}$ & 0.00947 & 0.00947 \\
\hline$(12,3,3)$ & 0.88502 & $\mathbf{0 . 0 1 0 0 6}$ & 0.00829 & 0.00829 \\
\hline
\end{tabular}

An implementation of this procedure is given for the two cases considered in Section 2.3, namely $\lambda_{1}=$ $\lambda_{2}=4, \mu=\mu_{0}=10$ and $\lambda_{1}=8, \lambda_{2}=1, \mu=10, \mu_{0}=12$. For the first case, we take $\left(c_{0}, c_{1}, c_{2}\right)=(2,1,1)$ while the allowed stock investment is $C=32$ (note that all cases listed in Table 2 represent a stock investment of 32). For this first case, the steps of the greedy procedure are as in Table 3. The procedure terminates with the stock allocation $\boldsymbol{S}=(13,3,3)$. From the experiments in Table 2, it is observed that when $S_{0}$ is greater than or equal to 11, the maximum fill rate is indeed obtained for the allocation $\boldsymbol{S}=(13,3,3)$. For Table 2, the reason for considering the allocations such that $S_{0} \geq 11$ is that 11 is the minimum $S_{0}$ value such that $F R\left(S_{0}, \infty, \infty\right) \geq F R(13,3,3) \approx 0.90514$ when $F R\left(S_{0}, \infty, \infty\right)$ is approximated as $\left(1-\rho_{0}^{S_{0}}\right)$ using the near-product-form steady state probability distribution. (Note that since we start the experiments in Table 2 with the allocation having the highest $S_{0}$ allowed by the available investment amount $C$, while making these experiments it is possible to consider the highest fill rate observed up to that point in time and compare it to $F R\left(S_{0}, \infty, \infty\right)$ with minimum $S_{0}$ required. This is basically the way to determine the minimum $S_{0}$ value considered in Table 2.) What is meant by the previous sentence is that even when there are always infinitely many items available at component stock points, having $S_{0}=10$ is not enough to beat the case $\boldsymbol{S}=(13,3,3)$ with fill rate 0.90514 . So, in order to check the optimality of $\boldsymbol{S}=(13,3,3)$ when $C=32$, in Table 2 there is no need to consider the allocations with $S_{0}=10$ and some finite $S_{1}, S_{2}$ values that would necessitate a total investment of 32 . Note that this first system is symmetric, so the cases $\left(S, R, S_{0}\right)$ and $\left(R, S, S_{0}\right)$ such that $S \neq R$ result in the same performance measures for the assembly stock point. For that reason, only one of such cases, either $\left(S, R, S_{0}\right)$ or $\left(R, S, S_{0}\right)$, is considered in Table 2.

For the second system, we take $\left(c_{0}, c_{1}, c_{2}\right)=(4,1,2)$ while the allowed stock investment is $C=65$ (again, note that all cases listed in Table 5 in the appendix represent a stock investment of 65). For this second case, the steps of the greedy procedure are as in Table 6 in the appendix. The final stock allocation obtained is $\boldsymbol{S}=(10,23,1)$. Analysis of the figures in Table 5 over all possible allocations with a total investment of 65 and $S_{0}$ being greater than or equal to 8 (where 8 is chosen as explained for the previous example problem, i.e., as the minimum $S_{0}$ value such that $F R\left(S_{0}, \infty, \infty\right) \geq F R(11,19,1) \approx 0.90363$, here 
$F R(8, \infty, \infty) \approx 0.90$ is very close to 0.90363 and considering approximation errors it is reasonable to have $\left.S_{0} \geq 8\right)$ shows that $\boldsymbol{S}=(11,19,1)$ is, in fact, the allocation giving the highest fill rate but the difference between both simulation and approximation values of $F R(11,19,1)$ and $F R(10,23,1)$ is very small. Although there is no guarantee of obtaining the best stock allocation with the use of the greedy procedure (as seen for this example problem), convergence to an allocation with a fill rate very close to the highest one is promising as for the performance of the greedy procedure. For the purpose of improving the solution obtained by the greedy procedure considering the possibility of convergence to such non-optimal points, one could proceed with a local neighborhood search around the solution obtained.

These two implementations of the greedy optimization procedure give an indication of the behavior of the fill rate as a function of $\boldsymbol{S}$, in spite of the fact that $F R(\boldsymbol{S})$ (even the approximate one proposed in this article) has not been studied analytically.

\section{Summary and possible extensions}

In this paper, we have analyzed a two-indenture repairable item system. The notion "two-indenture" indicates the two level product structure of an assembly, consisting of multiple components, each of which may fail. Component repair takes place in a repair facility, modeled as a product form queuing network, while subsequent assembly of the component in end-item occurs in an assembly department, again modeled as a product form queuing network. In order to pursue a high system availability, both ready-for-use components and assemblies are held in stock as well. Hence, initially failed items are replaced by ready-for-use ones, while the repaired items serve to replenish inventories. This combined queuing and inventory phenomenon makes the system hard to analyze. Based upon an aggregation step and subsequently a slight modification of some border transition rates, we have constructed an approximate model that can be completely analyzed and produces results that are very close to the ones of the original model (obtained by simulation). In particular, excellent approximations for key performance measures such as fill rates and work-in-process levels have been obtained. Based upon these results, we have in addition designed a greedy optimization approach to determine how a given target service level (fill rate) can be obtained against minimal cost, leading in addition to the best balance between stocks of components and assemblies.

The results of this paper can be extended in many ways. First of all, in a companion paper ([1]), we analyzed a single product two-echelon capacitated model. Here, failed products are initially returned to a local base which provides a ready-for-use item instead (taken from a local stock), while the failed item may be possibly repaired there, after which it replenishes the local stock. If repair is not possible locally, the item is sent to a central repair facility, which supplies a ready-for-use item from its own stock, to replenish the local base stock, while the failed item will be repaired and at last replenishes the central facility stock. The interesting situation is one with one central repair facility and multiple local base repair stations, each with their own stock points. All repair facilities are again modeled as product form networks. Hence, instead of the aborescent (assembly) structure, studied in the current paper, we dealt in [1] with an inverse aborescent (distribution) structure. It has been shown that an approximation similar to the one presented in the current paper, again leads to highly accurate results, allowing again for the construction of a very satisfactorily performing greedy optimization algorithm.

Having obtained the results in these two papers, an immediate challenge is of course to combine them and to come up with an approximate analysis and subsequent optimization procedure for general multi-echelon, multi-indenture repairable item systems, where each repair facility is modeled as a (finite capacity) queuing network. Hence, product structures may reveal more than two levels while also there may be more than two echelons. Finally, we wish to extend the model to multiple assembly types or different installations. We believe that in principle such an extension of models is possible; however, the extension is not straightforward. An important phenomenon to be studied is the occurrence of commonality (components or sub-assemblies 
that are used in multiple product types). Also, some items may be irrepairable, leading to disposal and subsequent procurement of a new one (possibly with a long procurement lead time).

Further extensions of the model include the assumption of a finite source (as opposed to the Poisson failure rates discussed so far). In particular, when a limited number of installations is under full responsibility of a repair organization, we may end up with a multi-echelon, multi-indenture structure that is embedded in an overall closed system (since in principle a fixed number of components, sub-assemblies and assemblies are in one of three stages, in repair at some facility, in stock as a ready-for-use item, or in use at an installation). Again, the challenge will be to determine whether similar approximation methods may work, and hence possibly an overall closed queuing network algorithm for the approximated model may yield satisfactory results. Since a variety of well performing approximate Mean Value Analysis procedures have been developed for analyzing also non-product form networks (see, e.g., Buzacott and Shanthikumar[2]), this even opens a way to deal with repair and assembly facilities with general (and type-dependent) processing time distributions.

An interesting variation of the model would come up when the assumption on the number of components that cause failure of the assembly (which is one in the paper) is relaxed to allow simultaneous request of more than one component upon failure of an assembly. This relaxation leads to the general assembly networks that are also applicable to manufacturing systems driven by the demand of industrial goods (such as machines and equipment) or customer goods (such as cars, electronic appliances and white goods) with the additional difficulties posed by the fork-join queues. These difficulties could be handled under additional assumptions of the independence of different component manufacturing processes, which seem to be justified under heavy load, a sufficient number of components manufactured in the same facility, or high base stock levels.

Another line of research concerns the use of the analysis framework to study the impact of investments in alternatively more stock or more capacity in the repair or assembly centers. This is a question of much broader importance than just in the context of maintenance. In manufacturing systems in particular, the question to invest in capacity or inventory to provide an adequate market response is extremely important, and determines ultimately the flexibility of a company. Qualitative considerations lead to typologies such as "Make and Assemble to Stock", "Make to Stock and Assemble to Order" or "Make and Assemble to Order", based on considerations such as stability of demand, diversity of either end-items or constituting components, and the size of internal lead times (e.g., in a situation with a limited number of components that can be used in a large variety of end-items, one often chooses for the Make to Stock and Assemble to Order mode, see also Zijm[13]). The models presented here allow for quantitative trade-off's between capacities and inventories and therefore can be extremely useful in making the ultimate choice. This however requires further research, in particular in modeling more general manufacturing systems (including, e.g., set-up times, machine failures, quality issues, see, e.g., Hopp and Spearmann[5]) as well as a further extension of the combined queuing and inventory models presented here. This will be the topic of future research.

\section{References}

[1] Z.M. Avșar and W.H.M. Zijm. Resource-Constrained Two-Echelon Inventory Models for Repairable Item Systems. Kluwer Academic Publishers special issue on "Analysis and Modelling of Manufacturing Systems", to appear.

[2] J.A. Buzacott and J.G. Shantikumar. Stochastic Models of Manufacturing Systems. Prentice-Hall, EnglewoodCliffs, NJ, 1993.

[3] A. Gupta and S.C. Albright. Steady-State Approximations for a Multi-Echelon Multi-Indentured RepairableItem Invcentory System. Naval Research Logistics, Vol 40, pp. 479-493, 1993. 
[4] W.H. Hausman and G.D. Scudder. Priority Scheduling Rules for Repairable Inventory Systems. Management Science, Vol. 28, pp. 1215-1232, 1982.

[5] J.J. Hopp and M.L. Spearmann. Factory Physics: Foundations of Manufacturing Management. Burridge, Irwin Professional Publishing, 1995.

[6] Y.J. Lee and P. Zipkin. Tandem Queues with Planned Inventories. Operations Research, 40:936-947, 1992.

[7] J.A. Muckstadt. A Model for a Multi-Item Multi-Echelon Multi-Indenture Inventory System. Management Science, Vol. 20, pp. 472-481, 1973.

[8] J.W. Rustenburg. A System Approach to Budget-Constrained Spare Parts Management. Ph.D. Thesis, Eindhoven University of Technology, Eindhoven, The Netherlands, 2000.

[9] J.W. Rustenburg and G.J. van Houtum and W.H.M. Zijm. Spare Parts Management for Technical Systems: Resupply of Spare Parts under Limited Budgets. IIE Transactions, 32 (10), pp. 1013-1026, 2000.

[10] G.D. Scudder and W.H. Hausman. Spares Stocking Policies for Repairable Items with Dependent Repair Times. Naval Research Logistics Quarterly, Vol. 29, pp. 303-322, 1982.

[11] C.C. Sherbrooke. METRIC: A Multi-Echelon Technique for Recoverable Item Control. Operations Research, Vol. 16, pp. 122-141, 1968.

[12] W.H.M. Zijm and Z.M. Avșar. Capacitated Multi-Indenture Inventory Models for Repairable Item Systems. Working paper, Bilkent University and University of Twente, 2001.

[13] W.H.M. Zijm. Towards Intelligent Manufacturing Planning and Control Systems. OR Spektrum 22, pp. 313-345, 2000. 
Appendix 
Table 4: EW for $\rho=9 / 10, \rho_{0}=9 / 12$

\begin{tabular}{|c|c|c|c|c|c|}
\hline$\left(S_{1}, S_{2}\right)$ & $E W_{\text {sim }}$ & Conf.Interval & Std.Dev.EW & $E W_{a p p}$ & error $_{E W}$ \\
\hline$\overline{(0,0)}$ & 12.00326 & $(11.97464,12.03188)$ & 0.013 & 11.99341 & 0.08 \\
\hline$(1,1)$ & 10.53398 & $(10.50682,10.56114)$ & 0.013 & 10.60465 & 0.67 \\
\hline$(3,3)$ & 8.59582 & $(8.57076,8.62088)$ & 0.012 & 8.73745 & 1.65 \\
\hline$(5,5)$ & 7.30985 & $(7.28580,7.33389)$ & 0.011 & 7.46473 & 2.12 \\
\hline$(7,7)$ & 6.36341 & $(6.34230,6.38451)$ & 0.010 & 6.50980 & 2.30 \\
\hline$(9,9)$ & 5.63892 & $(5.61991,5.65794)$ & 0.009 & 5.76798 & 2.29 \\
\hline$(15,15)$ & 4.28789 & $(4.27415,4.30162)$ & 0.006 & 4.36214 & 1.7 \\
\hline$(1,0)$ & 11.07340 & $(11.04477,11.10203)$ & 0.013 & 11.10459 & 0.28 \\
\hline$\overline{(3,0)}$ & 9.51119 & $\begin{array}{l}(9.48418,9.53820) \\
\end{array}$ & 0.013 & 9.61226 & 1.06 \\
\hline$\overline{(5,0)}$ & 8.29666 & $(8.27147,8.32184)$ & $\overline{0.012}$ & 8.43316 & 1.65 \\
\hline$(7,0)$ & 7.35326 & $\begin{array}{l}(7.32968,7.37684) \\
\end{array}$ & 0.011 & 7.50156 & 2.02 \\
\hline$(9,0)$ & 6.61802 & $(6.59517,6.64088)$ & 0.011 & 6.76550 & 2.23 \\
\hline$(15,0)$ & 5.23868 & $(5.22158,5.25578)$ & 0.008 & 5.36148 & 2.34 \\
\hline$(1,2)$ & 10.26721 & $(10.24061,10.29381)$ & 0.012 & 10.35471 & 0.85 \\
\hline$(3,2)$ & 8.72499 & $(8.69948,8.75051)$ & 0.012 & 8.86239 & 1.57 \\
\hline$\overline{(5,2)}$ & 7.53121 & $(7.50616,7.55626)$ & $\overline{0.012}$ & 7.68329 & 2.02 \\
\hline$(7,2)$ & 6.60380 & $(6.58119,6.62641)$ & 0.011 & 6.75169 & 2.24 \\
\hline$(9,2)$ & 5.88110 & $(5.86061,5.90160)$ & 0.010 & 6.01563 & 2.29 \\
\hline$(15,2)$ & 4.52150 & $(4.50626,4.53674)$ & 0.007 & 4.61160 & 1.99 \\
\hline$\overline{(1,4)}$ & 10.07368 & $(10.04722,10.10014)$ & $\overline{0.012}$ & 10.16734 & 0.93 \\
\hline$(3,4)$ & 8.53231 & $(8.50758,8.55705)$ & $\overline{0.012}$ & 8.67501 & 1.67 \\
\hline$\overline{(5,4)}$ & 7.34091 & $(7.31662,7.36520)$ & 0.011 & 7.49592 & 2.11 \\
\hline$(7,4)$ & 6.41701 & $(6.39538,6.43864)$ & 0.010 & 6.56431 & 2.30 \\
\hline$(9,4)$ & 5.69770 & $(5.67814,5.71725)$ & 0.009 & 5.82826 & 2.29 \\
\hline$(15,4)$ & 4.34686 & $(4.33237,4.36134)$ & 0.007 & 4.42423 & 1.78 \\
\hline$\overline{(1,6)}$ & 10.02719 & $(10.00105,10.05333)$ & $\overline{0.012}$ & 10.12059 & 0.93 \\
\hline$(3,6)$ & 8.48584 & $(8.46146,8.51022)$ & 0.011 & 8.62826 & 1.68 \\
\hline$\overline{(5,6)}$ & 7.29455 & $(7.27067,7.31843)$ & 0.011 & 7.44916 & 2.12 \\
\hline$(7,6)$ & 6.37099 & $(6.34981,6.39217)$ & 0.010 & 6.51756 & 2.30 \\
\hline$(9,6)$ & 5.65202 & $(5.63284,5.67121)$ & 0.009 & 5.78150 & 2.29 \\
\hline$(15,6)$ & 4.30266 & $(4.28866,4.31666)$ & 0.007 & 4.37748 & 1.74 \\
\hline$\overline{(1,20)}$ & $\overline{10.01211}$ & $(9.98612,10.03810)$ & $\overline{0.012}$ & $\overline{10.10523}$ & $\overline{0.93}$ \\
\hline$(3,20)$ & 8.47081 & $(8.44663,8.49499)$ & 0.011 & 8.61291 & 1.68 \\
\hline$(5,20)$ & 7.27951 & $(7.25586,7.30315)$ & 0.011 & 7.43381 & 2.12 \\
\hline$(7,20)$ & 6.35590 & $(6.33494,6.37686)$ & 0.010 & 6.50220 & 2.30 \\
\hline$(9,20)$ & 5.63704 & $(5.61810,5.65599)$ & 0.009 & 5.76615 & 2.29 \\
\hline$(15,20)$ & 4.28785 & $(4.27411,4.30158)$ & 0.006 & 4.36212 & 1.73 \\
\hline
\end{tabular}


Table 5: Fill Rate for $\rho=9 / 10, \rho_{0}=9 / 12$

\begin{tabular}{|c|c|c|c|c|c|c|c|}
\hline$\left(S_{0}, S_{1}, S_{2}\right)$ & $F R_{s i m}$ & $F R_{a p p}$ & error $_{F R}$ & $\left(S_{0}, S_{1}, S_{2}\right)$ & $F R_{\text {sim }}$ & $F R_{a p p}$ & error $_{F R}$ \\
\hline$(16,1,0)$ & 0.75357 & 0.75331 & 0.03 & $(10,15,5)$ & 0.87791 & 0.87422 & 0.42 \\
\hline$(15,5,0)$ & 0.81708 & 0.81550 & 0.19 & $(10,13,6)$ & 0.86068 & 0.85641 & 0.50 \\
\hline$(15,3,1)$ & 0.79427 & 0.79265 & 0.20 & $(10,11,7)$ & 0.83836 & 0.83343 & 0.59 \\
\hline$(15,1,2)$ & 0.75778 & 0.75573 & 0.27 & $(10,9,8)$ & 0.80980 & 0.80420 & 0.69 \\
\hline$(14,9,0)$ & 0.86027 & 0.85767 & 0.30 & $(10,7,9)$ & 0.77330 & 0.76715 & 0.80 \\
\hline$(14,7,1)$ & 0.84593 & 0.84345 & 0.29 & $(10,5,10)$ & 0.72666 & 0.72027 & 0.88 \\
\hline$(14,5,2)$ & 0.82238 & 0.81965 & 0.33 & $(10,3,11)$ & 0.66685 & 0.66092 & 0.89 \\
\hline$(14,3,3)$ & 0.78626 & 0.78347 & 0.35 & $(10,1,12)$ & 0.59045 & 0.58581 & 0.79 \\
\hline$(14,1,4)$ & 0.73599 & 0.73340 & 0.35 & $(9,29,0)$ & 0.87861 & 0.87479 & 0.44 \\
\hline$(13,13,0)$ & 0.88508 & 0.88423 & 0.10 & $(9,27,1)$ & 0.89169 & 0.88868 & 0.34 \\
\hline$(13,11,1)$ & 0.87943 & 0.87652 & 0.33 & $(9,25,2)$ & 0.89537 & 0.89285 & 0.28 \\
\hline$(13,9,2)$ & 0.86468 & 0.86158 & 0.36 & $(9,23,3)$ & 0.89388 & 0.89149 & 0.27 \\
\hline$(13,7,3)$ & 0.84132 & 0.83792 & 0.40 & $(9,21,4)$ & 0.88890 & 0.88629 & 0.29 \\
\hline$(13,5,4)$ & 0.80780 & 0.80428 & 0.44 & $(9,19,5)$ & 0.88085 & 0.87779 & 0.35 \\
\hline$(13,3,5)$ & 0.76301 & 0.75966 & 0.44 & $(9,17,6)$ & 0.86969 & 0.86602 & 0.42 \\
\hline$(13,1,6)$ & 0.70538 & 0.70239 & 0.42 & $(9,15,7)$ & 0.85501 & 0.85065 & 0.51 \\
\hline$(12,17,0)$ & 0.90157 & 0.89808 & 0.39 & $(9,13,8)$ & 0.83613 & 0.83100 & 0.61 \\
\hline$(12,15,1)$ & 0.8989 & 0.89579 & 0.35 & $(9,11,9)$ & 0.81205 & 0.80607 & 0.74 \\
\hline$(12,13,2)$ & 0.89029 & 0.88713 & 0.35 & $(9,9,10)$ & 0.78133 & 0.77450 & 0.87 \\
\hline$(12,11,3)$ & 0.87547 & 0.87200 & 0.40 & $(9,7,11)$ & 0.74208 & 0.73455 & 1.01 \\
\hline$(12,9,4)$ & 0.85348 & 0.84969 & 0.45 & $(9,5,12)$ & 0.69180 & 0.68398 & 1.13 \\
\hline$(12,7,5)$ & 0.82358 & 0.81947 & 0.50 & $\begin{array}{l}(9,3,13) \\
\end{array}$ & 0.62717 & 0.61999 & 1.15 \\
\hline$(12,5,6)$ & 0.78458 & 0.78033 & 0.54 & $(9,1,14)$ & 0.54430 & 0.53899 & 0.98 \\
\hline$(12,3,7)$ & 0.73453 & 0.73049 & 0.55 & $(8,33,0)$ & 0.84864 & 0.84442 & 0.50 \\
\hline$(12,1,8)$ & 0.67081 & 0.66732 & 0.52 & $(8,31,1)$ & 0.86849 & 0.86530 & 0.37 \\
\hline$(11,21,0)$ & 0.90442 & 0.90087 & 0.39 & $(8,29,2)$ & 0.87610 & 0.87366 & 0.28 \\
\hline$(\mathbf{1 1}, \mathbf{1 9}, \mathbf{1})$ & 0.90675 & 0.90363 & 0.34 & $(8,27,3)$ & 0.87746 & 0.87538 & 0.24 \\
\hline$(11,17,2)$ & 0.90280 & 0.89977 & 0.34 & $(8,25,4)$ & 0.87522 & 0.87314 & 0.24 \\
\hline$(11,15,3)$ & 0.89382 & 0.89059 & 0.36 & $(8,23,5)$ & 0.87044 & 0.86805 & 0.27 \\
\hline$(11,13,4)$ & 0.87970 & 0.87608 & 0.41 & $(8,21,6)$ & 0.86337 & 0.86048 & 0.34 \\
\hline$(11,11,5)$ & 0.85996 & 0.85586 & 0.48 & $(8,19,7)$ & 0.85390 & 0.85034 & 0.42 \\
\hline$(11,9,6)$ & 0.83390 & 0.82931 & 0.55 & $(8,17,8)$ & 0.84164 & 0.83729 & 0.52 \\
\hline$(11,7,7)$ & 0.80035 & 0.79533 & 0.63 & $(8,15,9)$ & 0.82591 & 0.82069 & 0.63 \\
\hline$(11,5,8)$ & 0.75742 & 0.75222 & 0.69 & $(8,13,10)$ & 0.80585 & 0.79966 & 0.77 \\
\hline$(11,3,9)$ & 0.70252 & 0.69763 & 0.70 & $(8,11,11)$ & 0.78030 & 0.77304 & 0.93 \\
\hline$(11,1,10)$ & 0.63257 & 0.62853 & 0.64 & $(8,9,12)$ & 0.74762 & 0.73934 & 1.11 \\
\hline$(10,25,0)$ & $\begin{array}{l}0.89685 \\
\end{array}$ & $\begin{array}{l}0.89322 \\
\end{array}$ & 0.41 & $(8,7,13)$ & 0.70586 & 0.69670 & 1.30 \\
\hline$(10,23,1)$ & 0.90426 & 0.90120 & 0.34 & $(8,5,14)$ & 0.65224 & 0.64273 & 1.46 \\
\hline$(10,21,2)$ & 0.90420 & 0.90144 & 0.30 & $(8,3,15)$ & 0.58309 & 0.57442 & 1.49 \\
\hline$(10,19,3)$ & 0.89944 & 0.89662 & 0.31 & $(8,1,16)$ & 0.49398 & 0.48797 & 1.22 \\
\hline$(10,17,4)$ & 0.89071 & 0.88753 & 0.36 & & & & \\
\hline
\end{tabular}


Table 6: Greedy procedure for $\rho=9 / 10, \rho_{0}=9 / 12$

\begin{tabular}{|c|c|c|c|c|}
\hline$\left(S_{0}, S_{1}, S_{2}\right)$ & $F R_{a p p}$ & $\Delta_{0}$ & $\Delta_{1}$ & $\Delta_{2}$ \\
\hline$(0,0,0)$ & 0 & $\mathbf{0 . 0 0 6 2 5}$ & 0 & 0 \\
\hline$(1,0,0)$ & 0.02500 & 0.01031 & $\mathbf{0 . 0 2 0 0 0}$ & 0.00125 \\
\hline$(1,1,0)$ & 0.04500 & 0.01406 & $\mathbf{0 . 0 1 6 0 0}$ & 0.00325 \\
\hline$(1,2,0)$ & 0.06100 & $\mathbf{0 . 0 1 7 4 6}$ & 0.01280 & 0.00565 \\
\hline$(2,2,0)$ & 0.13085 & 0.01739 & $\mathbf{0 . 0 2 4 9 6}$ & 0.00770 \\
\hline$(2,3,0)$ & 0.15581 & 0.01948 & $\mathbf{0 . 0 2 0 9 9}$ & 0.01026 \\
\hline$(2,4,0)$ & 0.17680 & $\mathbf{0 . 0 2 1 4 2}$ & 0.01761 & 0.01285 \\
\hline$(3,4,0)$ & 0.26247 & 0.01917 & $\mathbf{0 . 0 2 4 7 6}$ & 0.01323 \\
\hline$(3,5,0)$ & 0.28723 & 0.02029 & $\mathbf{0 . 0 2 1 2 8}$ & 0.01522 \\
\hline$(3,6,0)$ & 0.30851 & $\mathbf{0 . 0 2 1 3 5}$ & 0.01826 & 0.01715 \\
\hline$(4,6,0)$ & 0.39393 & 0.01825 & $\mathbf{0 . 0 2 2 2 5}$ & 0.01599 \\
\hline$(4,7,0)$ & 0.41618 & 0.01882 & $\mathbf{0 . 0 1 9 3 6}$ & 0.01727 \\
\hline$(4,8,0)$ & 0.43554 & $\mathbf{0 . 0 1 9 3 6}$ & 0.01683 & 0.01847 \\
\hline$(5,8,0)$ & 0.51298 & 0.01613 & $\mathbf{0 . 0 1 8 9 1}$ & 0.01640 \\
\hline$(5,9,0)$ & 0.53189 & 0.01638 & 0.01659 & $\mathbf{0 . 0 1 7 0 9}$ \\
\hline$(5,9,1)$ & 0.56608 & 0.01532 & $\mathbf{0 . 0 1 7 9 0}$ & 0.00676 \\
\hline$(5,10,1)$ & 0.58398 & 0.01540 & $\mathbf{0 . 0 1 5 7 8}$ & 0.00718 \\
\hline$(5,11,1)$ & 0.59975 & $\mathbf{0 . 0 1 5 4 9}$ & 0.01389 & 0.00758 \\
\hline$(6,11,1)$ & 0.66171 & 0.01259 & $\mathbf{0 . 0 1 4 2 8}$ & 0.00684 \\
\hline$(6,12,1)$ & 0.67599 & 0.01256 & $\mathbf{0 . 0 1 2 6 3}$ & 0.00706 \\
\hline$(6,13,1)$ & 0.68861 & $\mathbf{0 . 0 1 2 5 5}$ & 0.01116 & 0.00727 \\
\hline$(7,13,1)$ & 0.73881 & 0.01011 & $\mathbf{0 . 0 1 1 1 6}$ & 0.00640 \\
\hline$(7,14,1)$ & 0.74997 & $\mathbf{0 . 0 1 0 0 4}$ & 0.00990 & 0.00649 \\
\hline$(8,14,1)$ & 0.79013 & 0.00809 & $\mathbf{0 . 0 0 9 6 8}$ & 0.00569 \\
\hline$(8,15,1)$ & 0.79981 & 0.00800 & $\mathbf{0 . 0 0 8 6 1}$ & 0.00568 \\
\hline$(8,16,1)$ & 0.80842 & $\mathbf{0 . 0 0 7 9 1}$ & 0.00766 & 0.00568 \\
\hline$(9,16,1)$ & 0.84007 & 0.00634 & $\mathbf{0 . 0 0 7 3 8}$ & 0.00490 \\
\hline$(9,17,1)$ & 0.84744 & 0.00625 & $\mathbf{0 . 0 0 6 5 7}$ & 0.00485 \\
\hline$(9,18,1)$ & 0.85402 & $\mathbf{0 . 0 0 6 1 7}$ & 0.00586 & 0.00479 \\
\hline$(10,18,1)$ & 0.87869 & 0.00492 & $\mathbf{0 . 0 0 5 5 8}$ & 0.00409 \\
\hline$(10,19,1)$ & 0.88427 & 0.00484 & $\mathbf{0 . 0 0 4 9 8}$ & 0.00401 \\
\hline$(10,20,1)$ & 0.88925 & 0 & $\mathbf{0 . 0 0 4 4 5}$ & 0.00394 \\
\hline$(10,21,1)$ & 0.89370 & 0 & $\mathbf{0 . 0 0 3 9 7}$ & 0.00387 \\
\hline$(10,22,1)$ & 0.89766 & 0 & $\mathbf{0 . 0 0 3 5 4}$ & 0 \\
\hline & & & & \\
\hline
\end{tabular}

